\title{
Special announcement: Guidelines to the Practice of Anesthesia - Revised Edition 2013
}

\author{
Richard N. Merchant, MD
}

Received: 27 August 2012/Accepted: 21 September 2012/Published online: 6 October 2012

(c) Canadian Anesthesiologists' Society 2012

In this issue of the Journal, the Canadian Anesthesiologists' Society (CAS) is pleased to present the latest update to the CAS Guidelines to the Practice of Anesthesia - 2013. ${ }^{1}$ Initially developed in 1974 and published in 1975, these Guidelines were published in booklet form throughout the $1990 \mathrm{~s}$ and $2000 \mathrm{~s}$ and then published as a special article within the Journal since $2010 .^{2}$

The Guidelines were developed by the Committee on Standards and approved by the Board of the Canadian Anesthesiologists' Society. The Committee reviews features of the Guidelines on an ongoing basis and responds to the changing practice of the specialty of anesthesiology with updates to the recommendations presented therein. Some updates are relatively minor or reflect improvements in subtle inconsistencies that previously have escaped notice- for example, since the newer vapourizers do not use traditional "keyed" filling systems, rather than describe an acceptable anesthetic vapourizer as a "keyed" filling device, the description has been reworded to identify a requirement for an "agent-specific" filling system to ensure the vapourizer is filled with the correct agent.

Similarly, a small inconsistency edged its way into the use of the Ramsay sedation scale (RSS) to define care: RSS 1 - 3 would generally be considered "lighter" sedation, and RSS 4 - 6 would normally be considered "deeper" sedation; a small change was made to apply this terminology consistently.

Perhaps the following update has greater impact. We reviewed the guidelines for preoperative laboratory testing, which have not been modified for some years. In general, we

\section{R. N. Merchant, MD ( $\square)$}

Committee on Standards, Canadian Anesthesiologists' Society,

1 Eglinton Avenue East, Suite 208, Toronto, ON M4P 3A1,

Canada

e-mail: richard.merchant@ubc.ca recognize that often too much laboratory testing is done without supporting evidence (likely to avoid cancelling or delaying surgery due to "missing" test results). The CAS Guidelines are fairly precise in this regard, but we did recognize that current thought would not consider age alone or isolated (treated) hypertension as specific indications for preoperative electrocardiogram (ECG) testing; therefore, we have removed those items from the diagnoses listed for ECG testing.

We recently presented a statement on respiratory monitoring of patients ${ }^{3}$ receiving sedative medications. Similar concerns regarding locations outside the operating room (OR) have not previously been addressed, hence the addition of the statement, "Respiratory monitoring should be considered in non-OR locations (postanesthesia care unit and elsewhere) for sedated patients and those at risk of respiratory depression".

Lastly, in light of the new CAS Position Paper on Procedural Sedation, we have received several questions about details of the somewhat general statements within that document. In response to those concerns and in recognition that the ECG had been mandated as a monitor of cardiac rhythm prior to the availability of any other high-quality monitor of cardiac function, we have added the statement, "Electrocardiogram monitoring may be waived in suitable patients when continuous pulse oximetry is used for minor procedures when only RSS 1 - 3 sedation is employed".

Those familiar with other guideline statements and other patient safety issues will agree with our concern that more work remains in the ongoing process of revision of our current Guidelines and development of new guideline principles. In particular, the Committee continues to examine issues in the areas of obstetrical anesthesia and analgesia, acute pain management, and clinician fatigue and competence. The CAS Committee on Standards and 
partner groups within the CAS continue to consider these and other issues, and they would gratefully receive any feedback offered by members of the Canadian anesthesia community.

\section{Communiqué spécial: Directives pour la pratique de l'anesthésie - Édition révisée 2013}

Dans ce numéro du Journal, la Société canadienne des anesthésiologistes (SCA) a le plaisir de présenter la dernière mise à jour des Directives de la SCA pour la pratique de l'anesthésie - 2013. ${ }^{1}$ Tout d'abord élaborée en 1974 et publiée en 1975, ces Directives ont été publiées sous forme de brochures au cours des décennies1990 et 2000, puis dans un article spécial du Journal depuis 2010. ${ }^{2}$

Les Directives ont été élaborées par le Comité des normes et approuvées par le conseil d'administration de la Société canadienne des anesthésiologistes. Le Comité étudie en permanence le contenu des Directives et répond à l'évolution de la pratique de l'anesthésiologie par des mises à jour et des recommandations qui sont présentées ici. Quelques mises à jour sont relativement mineures ou sont le reflet d'améliorations pour des incohérences mineures qui avaient échappé précédemment à l'attention du Comité. Considérant, par exemple, que les vaporisateurs les plus récents n'utilisent pas les systèmes de remplissage traditionnels à clés,la description d'un vaporisateur convenable a été reformulée pour identifier la nécessité de disposer d'un système de remplissage spécifique pour chaque agent (pour s'assurer que le vaporisateur est rempli avec le bon médicament) plutôt que de mentionner un dispositif de remplissage « à clef ».

De même, une petite incohérence s'est glissée dans l'utilisation de l'échelle de sédation de Ramsay (RSS) pour la définition des soins: RSS 1 - 3 seraient généralement considérés comme une sédation « légère » et RSS 4 - 6 seraient normalement considérés comme une sédation « profonde ». Une petite modification a été apportée pour rendre cette terminologie plus cohérente.

La mise à jour suivante a peut-être un impact plus important: Nous avons revu les directives concernant les examens de laboratoire préopératoires, qui n'avaient pas été modifiées depuis quelques années. D'une manière générale, nous reconnaissons que, souvent, trop de tests de laboratoire sont effectués sans raisons particulières (susceptibles d'entrainer l'annulation ou le reportd'une intervention à cause du résultat «manquant» des tests). Les Directives de la SCA sont assez précises dans ce domaine mais nous avons reconnu que la pensée actuelle ne considérerait pas l'âge seul ou une hypertension (traitée) isolée comme des indications spécifiques pour exiger un électrocardiogramme (ECG) préopératoire; nous avons donc supprimé ces points de la liste des diagnostics pour lesquels un ECG est justifié.

Nous avons récemment présenté une mise au point sur la Surveillance de la respiration des patients ${ }^{3}$ recevant des sédatifs. Des préoccupations similaires n'ont pas été abordées antérieurement concernant des emplacements hors de la salle d'opération; aussi, la mise au point sur la «Surveillance de la respiration doit être prise en compte dans un contexte hors salle d'opération (salle de réveil et ailleurs) pour les patients ayant reçu une sédation et pour ceux qui présentent un risque de dépression respiratoire ».

Enfin, à la lumière du nouvel Exposé de principe sur la sédation consciente de la SCA, nous avons reçu plusieurs questions concernant des détails des énoncés plutôt généraux contenus dans ce document. En réponse à ces préoccupations et reconnaissant que l'ECG a été rendu obligatoire pour le monitorage du rythme cardiaque avant que ne deviennent disponibles d'autres moniteurs de grande qualité de la fonction cardiaque, nous avons ajouté l'énoncé suivant ${ }^{\circ}$ : "la surveillance à l'aide de l'électrocardiogramme peut être omise chez les patients sélectionnés quand une oxymétrie de pouls continue est utilisée pour des interventions mineures faisant appel uniquement à une sédation RSS 1 - 3 ».

Ceux qui ont l'habitude des autres énoncés des Directives et des autres problèmes de sécurité des patients seront d'accord avec nous pour dire qu'il reste encore beaucoup de travail à faire sur le processus de révision en cours de nos Directives actuelles et pour l'élaboration de nouveaux principes dans ces directives. Le Comité continue notamment à examiner les problèmes posés dans les domaines de l'anesthésie et de l'analgésie obstétricales, de la prise en charge de la douleur aiguë, de la fatigue et de la compétence des cliniciens. Le Comité des normes de la SCA et les groupes partenaires au sein de la SCA continuent d'étudier ces problèmes et d'autres, et ils seront heureux de recevoir un retour d'information de la part des membres de la communauté des anesthésiologistes canadiens.

Competing interests None declared.

\section{References}

1. Merchant $R$, Chartrand D, Dain S, et al. Guidelines to the Practice of Anesthesia - Revised Edition 2013. Can J Anesth 2013; 60: this issue.

2. Merchant RN. Special announcement: Guidelines to the practice of anesthesia - Revised edition 2010 (Editorial). Can J Anesth 2010; 57: $15-7$

3. Kurrek MM, Merchant R. Yesterday's luxury - today's necessity: end-tidal $\mathrm{CO}_{2}$ monitoring during conscious sedation. Can J Anesth 2012; 59: 731-5. 